\title{
A Rare Case of Intra Articular Osteoid Osteoma of Hip Joint
}

\author{
Dr.Krishna Prasad Nimmagadda ${ }^{1}$, Dr.V.Somanatham ${ }^{2}$, Dr. Saketh Kolla ${ }^{3}$, \\ ${ }^{1,2,3}$ (Dept of Orthopaedics, Dr. PSIMS \& RF, Gannavaram, Krishna Dt)
}

\begin{abstract}
Osteoid osteoma accounts for 10\% of all benign bone tumours affecting young adults of age group 10-30 years. Intra articular osteiod osteoma accounts for approximately $13 \%$ of all osteoid osteoma cases and present as monoarthropathy. Clinical and radiological features of osteiod osteoma are atypical when located intra articularly and differ significantly from extra articular lesions. Intraarticular osteoid osteoma presents a diagnostic challenge and can cause a delay in diagnosis for years. We present a case of 39 year old female with a rare Intraarticular osteoid osteoma of hip involving femoral head diagnosed early with CT imaging and bone scintigraphy. Complete excision of the tumour with femoral head and hemiarthroplasty with modular bipolar prosthesis was done and diagnosis was confirmed by histopathological examination. Hence a high index of suspicion with correlation between clinical and appropriate radiological imaging is necessary for early diagnosis and effective management of the tumour.
\end{abstract}

Key Words: Osteoid osteoma, Intra articular, hip.

\section{Introduction}

Osteoid osteoma is a benign osteoblastic tumour usually occurs in young adults(10-30 years).Nocturnal pain,response to NSAIDs,radiolucent nidus with surrounding sclerosis are its characteristic features. When located intra articularly, the typical features of osteoid osteoma are absent thus leading to difficulty in diagnosis, delay in appropriate management. We report a case of Intraarticular osteoid osteoma hip in whom diagnosis was made early using advanced imaging like CT(fig.2,fig.3), bone scan(fig.4.) and effectively managing the case with complete excision of tumour and femoral head along with modular bipolar hemiarthroplasty of left hip.

\section{Case Report}

A 39 year old female patient presented with complaints of pain in left hip for the last 15 years, insidious in onset, aggravated since 2 years. The general examination of patient is normal. All Left Hip movements are painful and restricted. Blood investigations $-\mathrm{Hb}$, Total leukocyte count, differential count, ESR, CRP etc are all within normal range. The patient is a known diabetic and is on regular oralhypoglycemics.

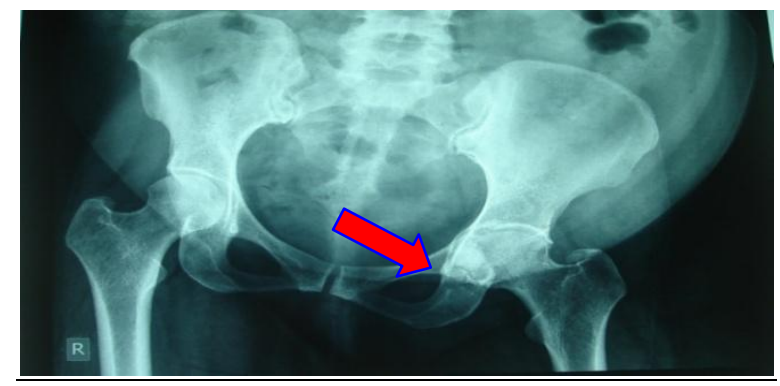

Fig.1.

$\underline{\mathrm{X} \text {-ray }}$ : pelvis with both hips: Shows sclerosis at head of left femur \& pelvic obliquity.

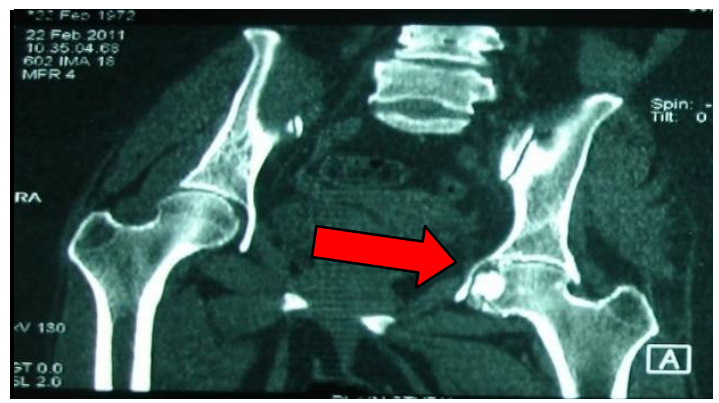

Fig 2 
: CT scan: Hyperdense irregular lesion of bony attenuation seen arising from the head of left femur near fovea capitis adjoining the medial end of acetabulum

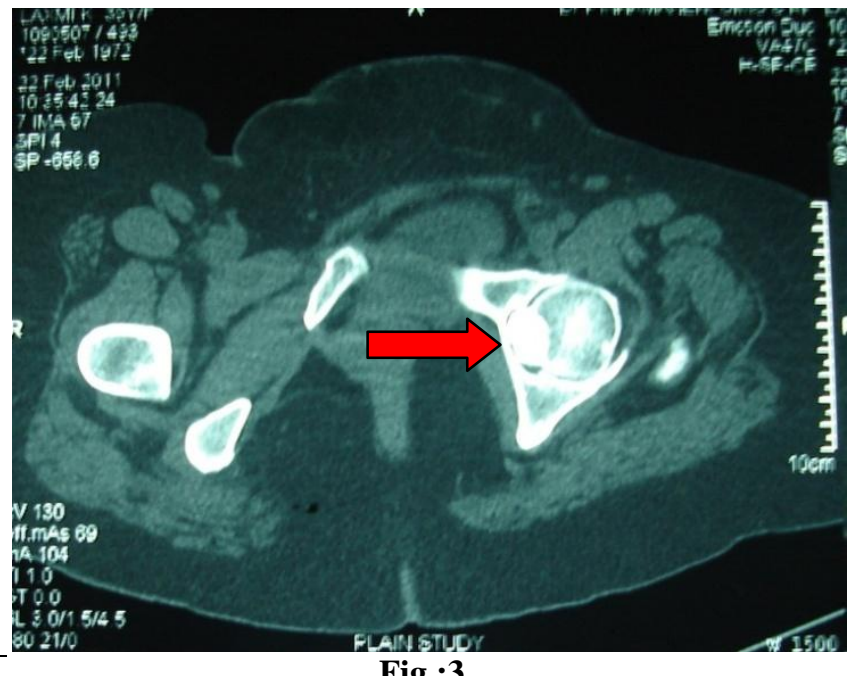

Fig :3

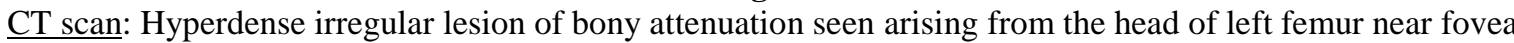
capitis adjoining the medial end of acetabulum.

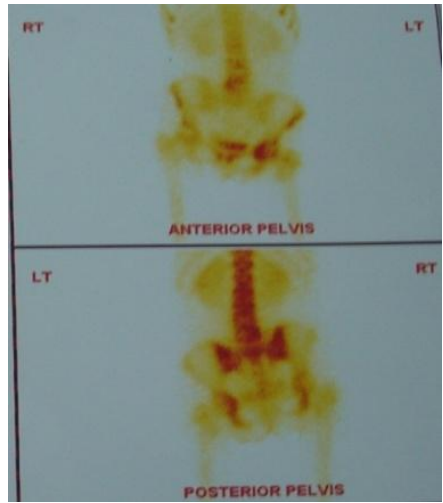

Fig: 4

Bone scan: Increase in tracer uptake in left femur and acetabulum is seen.(Fig.4.)

\section{Aspiration Biopsy-Inconclusive}

Surgical management, posterior approach-tumour along with head of the femur is excised completely (Fig.5.) and hemi arthroplasty with modular bipolar prosthesis left hip (Fig.6, Fig.7.) is performed and the excised tumour along with the head of femur is sent for histopathological examination which confirmed the diagnosis of osteoid osteoma (Fig.8.).Post operative period is uneventful and no evidence of any recurrence on follow ups for 3years.
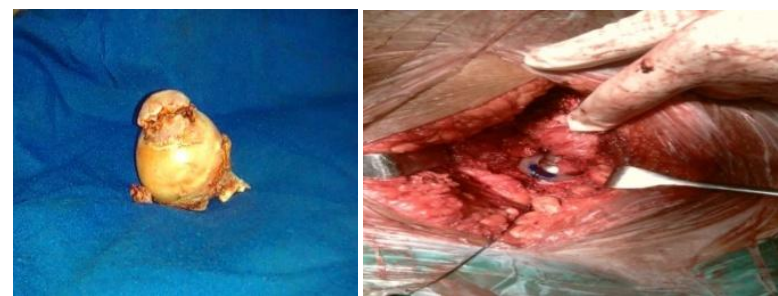
Fig.5. Gross section

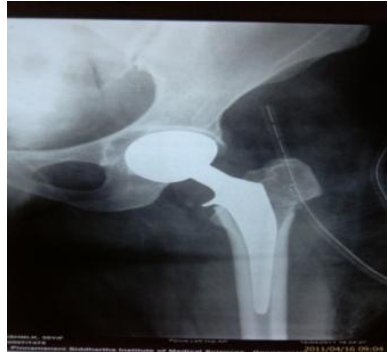

Fig.7. Post op-X ray
Fig.6. Intra operative

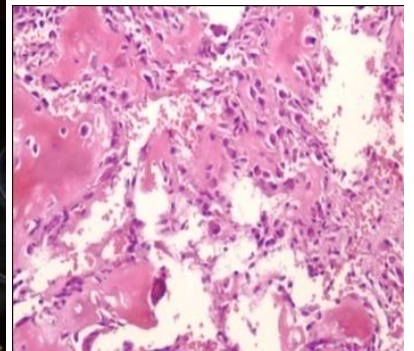

Fig.8.Microscopy

\section{Discussion}

Osteoid osteoma is a benign bone lesion of uncertain origin that accounts for approximately $10 \%$ of all benign bone tumors. Osteoid osteoma rarely occurs after 30 years of age. The typical location is the subperiosteal region of the diaphysis of long bones, most frequently the femur followed by the tibia. Osteoid osteomas have been categorized by location as subperiosteal, cortical, and cancellous. Intra-articular osteoid osteoma accounts for approximately $13 \%$ of all osteoid osteomas, the commonest site being the hip. The ankle, elbow, wrist and knee are affected less commonly and presents as a monoarthropathy and presents diagnostic difficulties, which are important for orthopedic surgeons. The features differ significantly from the well known classical hallmarks of the extra-articular lesion. The radiological and clinical findings are uncharacteristic and misleading, and the lesions are difficult to identify. Clinical presentation of intra-articular lesions typically consists of variable articular pain indistinguishable from other arthropathies. Unlike the more classically located osteoid osteoma, night pain is not typical. Also, the pain from intra-articular lesion is less responsive to nonsteroidal anti-inflammatory drugs than the extra-articular variety Joint tenderness, soft tissue swelling, synovitis and effusion are typical, like any mono-articular inflammatory arthritis .It has been shown that the level of prostaglandin E2 (PGE2) and prostacyclin (PGI2) can reach levels ten times their production in normal tissue. In intraarticular osteoid osteoma, these mediators of inflammation may play an important role in the pathogenesis of the concomitant synovitis. The diagnosis of an intraarticular osteoid osteoma is often delayed for months or years. Patients present with a painful joint that may be warm and stiff, suggesting synovitis. Synovitis associated with intraarticular osteoid osteomas is often hyperplasic.

The classic radiographic appearance of the more common cortical osteoid osteoma is that of a radiolucent nidus, with or without internal calcification and surrounding sclerosis or cortical thickening. This is in contrast to intraarticular osteoid osteomas, which are characterized by little or no reactive sclerosis; for this reason, the radiolucent nidus is often overlooked on radiographs. Although endosteal sclerosis may occur with intraarticular osteoid osteomas, periosteal new bone is absent, because the periosteum is not present within the joint capsule The subtle radiographic appearance combined with a nonspecific clinical presentation often leads to delay of diagnosis and further evaluation using advanced imaging studies. Bone scintigraphy three-phase technetium-99m bone-scan and computed tomography (CT) are essential for an accurate and early diagnosis. These imaging techniques reveal abnormalities in bone and cartilage growth, new bone formation and sclerosis distant from the tumour on either side of the joint, and disruption of the articular surface. Radiological follow up after surgery shows that these changes can be reversible, especially when the diagnosis is made early. CT and MR imaging findings of osteoid osteoma are well known. On CT, an osteoid osteoma is characterized by a lowattenuation nidus with possible internal calcification and variable surrounding sclerosis. On MR imaging, the nidus shows low or intermediate signal on T1-weighted images, variable signal on T2-weighted images, and variable contrast enhancement after IV gadolinium administration. In addition, high signal in the bone marrow and soft-tissue abnormalities on T2-weighted images may be found adjacent to the osteoid osteoma.

Technetium bone scanning has high sensibility but low specificity. It commonly shows increased uptake centrally that distinguishes the lesion from osteomyelitis or abscess. This sign is characterized by a focal hot spot of the nidus and an area of low peripheral radionuclide accumulation around it, which is related to the sclerotic bone (double density sign) typically absent in intra articular osteoid osteomas. Focal accumulation of the radioisotope is commonly seen, corresponding to location of the nidus.

Differential diagnosis: These include acute, sub acute and chronic inflammatory arthritis like septic or tuberculous arthritis, a vascular necrosis of femoral head, pigmented villonodular synovitis, synovial chondromatosis, gout, rheumatoid arthritis, osteoarthritis, Brodie's abscess and intracortical chondroblastoma. Modalities of treatment included Open surgery for excision of tumour, CT-guided ablation using radiofrequency (RF) and arthroscopic surgery. In our case, open surgery for excision of the tumour with the femoral head and 
hemiarthroplasty with modular bipolar prosthesis was opted for preventing recurrence of tumour.The tumour has been excised completely to prevent recurrence and histopathological examination confirmed the same.

\section{Conclusion}

In conclusion, the features of intra articular osteoid osteoma differ from extra articular osteiod osteoma posing difficulty in diagnosing the tumour. So a high index of suspicion clinically and radiological association with advanced imaging like C.T and Bone Scintigraphy should be done for early diagnosis and effective management of intra articular osteoid osteoma.

\section{References}

[1]. Miller SJ, Smith DW, Beckett WW, Gaines RW. Intra-articular osteoid osteoma of the acetabulum in a 6 year old. Orthopedics. 1997;20(7):654-5.

[2]. Pikoulas C, Mantzikopoulos G, Thanos L, Passomenos D, Dalamarinis C, Glampedaki-Dagianta K. Unusually located osteoid osteomas. Eur J Radiol. 1995;20(2):120-5.

[3]. Norman A, Abdelwahab IF, Buyon J, Matzkin E. Osteoid osteoma of the hip stimulating an early onset of osteoarthritis. Radiology. $1986 ; 158(2): 417-20$.

[4]. Allen SD, Saifuddin A. Imaging of intra-articular osteoid osteoma. Clin Radiol. 2003;58(11):845-52.

[5]. Cassar-Pullicino VN, McCall IW, Wan S. Intra-articular osteoid osteoma. Clin Radiol. 1992 Mar; 45(3):153-160.

[6]. Greco F, Tamburrelli F, Ciabattoni G. Intra-articular osteoid osteoma. Ital J Orthop Traumatol. 1992; 18(1):63-

[7]. 69.

[8]. Goldman AB, Schneider R, Pavlov H. Osteoid osteomas of the femoral neck: report of four cases evaluated with isotopic bone scanning, CT, and MR imaging. Radiology. 1993;186(1):227-32.

[9]. Alvarez MS Moneo PR, Palacios JA. Arthroscopic extirpation of an osteoid osteoma of the acetabulum. Arthroscopy. 2001;17(7):768-71.

[10]. Lee DH, Jeong WK, Lee SH. Arthroscopic excision of osteoid osteomas of the hip in children. J Pediatric Orthop. 2009;29(6):54751. 